\title{
Inteligencias múltiples y su desarrollo en los procesos pedagógicos, una revisión sistemática
}

\section{Multiple intelligences in pedagogical processes, a systematic review}

1 Jeanneth Caroline Galarza Galarza

Universidad Técnica de Ambato, Facultad de Ciencias Humanas y de la Educación, Carrera de Educación Inicial-Educación Básica. Ambato, Ecuador, Doctorando en Ciencias de la Educación Universidad Nacional de Trujillo, Perú.

jeannethcgalarzag@uta.edu.ec

2 Milena Aracely Estupiñán Guamani (iD) https://orcid.org/0000-0002-5874-5764 Universidad Técnica de Ambato, Facultad de Ciencias Humanas y de la Educación, Carrera de Educación Inicial. Ambato, Ecuador, ma.estupinan@uta.edu.ec

$3 \quad$ Silvia Beatriz Acosta Bones i https://orcid.org/0000-0002-5874-5764 Universidad Técnica de Ambato, Facultad de Ciencias de la Educación, Carrera de Educación Inicial. Ambato, Ecuador.

silviabacostab@uta.edu.ec

4 Elena del Rocío Rosero Morales (iD) https://orcid.org/0000-0001-7004-3207

Universidad Técnica de Ambato, Facultad de Ciencias Humanas y de la Educación,

Carrera de Educación Inicial. Ambato, Ecuador.

elenadroserom@uta.edu.ec

Artículo de Investigación Científica y Tecnológica

Enviado: 24/12/2021

Revisado: 29/12/2021

Aceptado: $12 / 01 / 2022$

Publicado:08/03/2023

DOI: https://doi.org/10.33262/concienciadigital.v6i1.4.1995

Cítese: Galarza Galarza, J. C., Estupiñán Guamani, M. A., Acosta Bones, S. B., \& Rosero Morales, E. del R. (2023). Inteligencias múltiples y su desarrollo en los procesos pedagógicos, una revisión sistemática . ConcienciaDigital, 6(1.4), 233-250. https://doi.org/10.33262/concienciadigital.v6i1.4.1995

CONCIENCIA DIGITAL, es una Revista Multidisciplinar, Trimestral, que se publicará en soporte electrónico tiene como misión contribuir a la formación de profesionales competentes con visión humanística y crítica que sean capaces de exponer sus resultados investigativos y científicos en la misma medida que se promueva mediante su intervención cambios positivos en la sociedad. https://concienciadigital.org

La revista es editada por la Editorial Ciencia Digital (Editorial de prestigio registrada en la Cámara Ecuatoriana de Libro con No de Afiliación 663) www.celibro.org.ec

Esta revista está protegida bajo una licencia Creative Commons Attribution Non Commercial No Derivatives 4.0 International. Copia de la licencia: $\underline{\text { http://creativecommons.org/licenses/by-nc-nd/4.0/ }}$ 


\section{Palabras}

claves:

inteligencias

múltiples, pedagogía, didáctica, aprendizaje, desarrollo cognitivo

\section{Keywords:}

multiple intelligences, pedagogy, didactics, learning, cognitive development

\section{Resumen}

La Teoría de las Inteligencias Múltiples aparece en el mundo de la Psicología en 1983, luego se expande a otras disciplinas. Edward Gardner expone en base a varios estudios, la existencia no de una sino de ocho inteligencias diferentes que se desarrollan en los dos hemisferios cerebrales. Esta teoría potencia el análisis, estudios e investigaciones sobre su aplicación en los procesos de enseñanza aprendizaje, que engloba a todo el mundo. La pedagogía aplicada en los diferentes modelos educativos potencia aún más el estudio de la relación con las inteligencias diferenciadas y a la vez conectadas entre sí. El objetivo del estudio se centra en actualizar información sobre la aplicación de la Teoría de las Inteligencias Múltiples con el proceso de enseñanza aprendizaje; para lo que se aplica la metodología cualitativa, mediante la revisión documental y bibliográfica en las bases de datos regionales Latindex, Scielo y Redalyc a partir de palabras clave como: Inteligencias múltiples, pedagogía, didáctica, aprendizaje, desarrollo cognitivo; la búsqueda se realiza en idioma español. De entre los artículos encontrados se seleccionaron nueve que muestran que los estudios relacionados con las inteligencias múltiples y su relación directa con la pedagogía en sus diferentes formas.

\section{Abstract}

The Theory of Multiple Intelligences first appeared in the world of Psychology in 1983, and then expanded to other disciplines. Its mentalize is Edward Gardner who exposes, based on several studies, the existence of not one but eight different intelligences that develop in the two cerebral hemispheres. This theory enhances the analysis, studies and research on its application in the teaching-learning process, which encompasses everyone. The pedagogy applied in the different educational models further enhances the study of the relationship with differentiated intelligences and at the same time connected to each other. The objective of the study focuses on updating information on the application of the Theory of Multiple Intelligences with the teaching-learning process; For which the qualitative methodology is applied, through the documentary and bibliographic review in the regional databases Latindex, Scielo and Redalyc from keywords such as: Multiple intelligences, pedagogy, didactics, 
learning, cognitive development; The search is carried out in Spanish. Among the articles found, nine were selected that show that studies related to multiple intelligences and their direct relationship with pedagogy in its different forms.

\section{Introducción}

Howard Gardner, impulsa su teoría de las inteligencias múltiples cuestionando que ésta es un todo global y único y argumentando el funcionamiento a partir de una serie de partes, cada una con sus particularidades que las definen y que les permiten desarrollar unas sobre otras. Las inteligencias múltiples sin duda tienen conexión directa con el aprendizaje, de allí que se estudian desde el enfoque de las habilidades y capacidades de los seres humanos, es decir, con la parte funcional de la pedagogía y de los modelos educativos, en especial del holístico (Chura, 2019).

La inteligencia múltiple engloba a todo, es decir, desde la importancia de ser inteligente y formar parte activa en la capacidad de decidir, las relaciones sociales o deportes; sin duda, se refieren al desarrollo de habilidades para resolver problemas o dificultades (Chilig, 2018).

De acuerdo con Gardner en los seres humanos hay las inteligencias: lingüística-verbal, lógica- matemática, intrapersonal, interpersonal, corporal-cinestésica, musical, naturalista, espacial. se habla de la novena inteligencia que vendría a ser la existencial, sin embargo, aún no está validada.

Se debe tener en cuenta las siguientes señales. Según Gardner (2001)

1. La identificación de la «morada» de la inteligencia por daño cerebral.

2. La existencia de individuos excepcionales en ámbitos específicos de la solución de problemas o de la creación.

3. El gatillo neural preparado para dispararse en determinados tipos de información interna o externa.

4. La susceptibilidad a la modificación de la inteligencia mediante entrenamiento.

5. Una historia de plausibilidad evolutiva.

6. Los exámenes específicos mediante tareas psicológicas experimentales.

7. El apoyo de exámenes psicométricos.

8. La creación de un sistema simbólico específico. 


\section{Inteligencia lingüística}

En ella intervienen los dos hemisferios del cerebro, se llama también inteligencia lenguaje. Según Prado (2017) "un niño se expresa con mayor frecuencia a través de la palabra, ama comunicarse, leer, escribir, contar, ama exponer utilizando el lenguaje en forma oral o escrita, usando de manera eficiente las estructuras lingüísticas" (p. 272).

Este tipo de inteligencia está inmersa en el mundo del aprendizaje desde sus primeras etapas; se basa en cuatro componentes dentro del cuerpo de los currículos vigentes. Es tal la importancia de la inteligencia lingüística, hoy en día que es evaluada con altos indicadores dentro de las habilidades básicas que son requisitos para ingresar a la universidad.

\section{Inteligencia musical}

Uno de los componentes de esta inteligencia es tener buen oído y están inmersos en actividades de producción musical sean cantantes o bailarines. Aparece desde la génesis y diferente entre las personas. Las características son: Tener bases artísticas como la creatividad, concentración, resolución de problemas, auto eficiencia, coordinación y desarrolla la atención y la autodisciplina

Según Jensen (2010) el cerebro cuenta con las capacidades para entender y diseñar música y cada vez se hace más temprano, es decir, desde que está en el vientre de la madre y luego el entorno favorecer puede llegar a dar grandes resultados, tal como revela la historia del músico universal Wolfgang Amadeus Mozart.

\section{Inteligencia lógico-matemática}

Consiste en observar, percibir, hacer series, sacar conclusiones, manejar algoritmos y sobre todo solucionar problemas. Funciona mucho más el hemisferio izquierdo del cerebro y pueden dedicarse a las ciencias exactas. "La atención a estudiantes con capacidades excepcionales debe realizarse desde edades tempranas, puesto que les permite contar con mayores oportunidades de sobresalir y sentirse a gusto trabajando durante ellas, además de servir de impulso para su vida profesional" (Valbuena et al., 2018, p.68).

El desarrollo de esta inteligencia es muy bien vista desde el ejemplo más simbólico de Albert Einstein, un alumno poco destacado en la primaria y secundaria mientras, que en la aplicación diaria resultó el creador de varias teorías matemáticas.

En la aplicación de la inteligencia matemática se la asocia con las personas de coeficiente mucho mayor que el promedio y quienes no la desarrollan pues, automáticamente son calificados como menos "aptos". La inteligencia matemática también es parte componente esencial de las pruebas genéricas de aprendizaje. 


\section{Inteligencia espacial o visual}

Esta inteligencia la tienen las personas que puede hacer un modelo mental en tres dimensiones del mundo en su defecto extraer un fragmento de él. Los seres humanos desarrollan observaciones personales del mundo visual, es decir, más allá de lo fisiológico, por ejemplo, un ciego podría desarrollar inteligencia visual (Gardner 2001).

En esa consideración, la inteligencia espacial no está asociada directamente con un sentido, sino que está relacionada con varios otros factores, como los sociales y emocionales. Esta inteligencia se desarrolla en el hemisferio derecho. La aplicación del desarrollo de esta inteligencia se ha considerado dentro de la práctica docente, cuando a los niños les enseñan desde la ubicación en sus diferentes contextos, es decir, en los rincones de aprendizaje.

\section{Inteligencia corporal - kinestésica}

Se relaciona con la habilidad que tiene el individuo para mover el cuerpo. La inteligencia corporal o kinestésica se evidencia a partir de la motricidad fina y gruesa.

Este tipo de inteligencia es la capacidad del cuerpo para desarrollar algunos movimientos o actividades, así como crear o resolver conflictos (Nadal, 2015), esto nos indica que los individuos que desarrollan esta inteligencia lo hacen a través de procesos táctiles, los mismos que sirven para comprender.

Se relaciona, además, con actividades como la danza, gimnasia o incluso atletismo. Dentro de este tipo se encuentran los bailarines, cirujanos y deportistas, en que la kinesia se manifiesta principalmente, desde cuando son niños.

\section{Inteligencia intrapersonal}

Se refiere al desarrollo del "yo interno" con la capacidad de reflexionar y evaluar los diferentes aspectos, sentimientos, acciones, es decir, conocerse a sí mismo. Según Mena et al. (2020), el individuo reflexiona y es consciente de las emociones; aspectos que están localizados en los lóbulos temporal y frontal que finalmente, desarrollan empatía.

\section{Inteligencia interpersonal}

Llamada también personal, es estudiada desde la teoría del psicoanálisis y da cuenta de la relación consigo mismo y los demás. Desarrolla la habilidad de comunicarse y laborar con otras personas; Los ejemplos más palpables son disertaciones efectuadas por religiosos, terapeutas, políticos y hoy en día las personas vinculadas con las ventas (Ernest-Slavit, 2001). 
En la actualidad, el individuo la desarrolla con mayor facilidad porque se desarrollan actividades que implican relaciones más directas, por ejemplo, las ventas que dan paso a subdisciplinas como el estudio de las relaciones humanas (Goleman, 1996).

\section{Inteligencia naturalista-pictórica}

Se desarrolla al observar y estudiar la naturaleza, los biólogos son quienes más la han desarrollado. También resalta el desarrollo de las cualidades pictóricas del individuo, por su relación con su capacidad de observar, interpretar y reproducir lo que ve el pintor/escultor/diseñador.

Se refiere a la habilidad de reconocer flora y fauna; animales o el ambiente en general, así como la posibilidad de explorar y entender el mundo natural y usar los conocimientos para mejorar cultivos y desarrollar todo lo relacionado con ciencias biológicas.

Según Gardner (2001), el individuo, además, de la capacidad de "solucionar inconvenientes, crear productos de costo en una o más civilizaciones", de esa manera concluye que el cerebro no tenía el estado de madurez al mismo nivel que el cuerpo.

En este apartado también se estudia la inteligencia relacionada con el coeficiente intelectual (CI). Con el conocimiento sobre las inteligencias se puede comprender la relación entre lo que se aprende con la toma de decisiones y resolver problemas, sin embargo, esto dependería de qué tipo de inteligencia tiene cada persona, también se pueden desarrollar las que obtuvimos menos desarrolladas para lograr tener otras inteligencias (Ramírez, 2018).

\section{Bases teóricas de la pedagogía}

La pedagogía, considerada como la ciencia de la educación y la enseñanza, está compuesta por saberes teóricos y filosóficos que permiten el desarrollo y construcción del estudiante. Funciona a partir del manejo de técnicas y métodos de aprendizaje que conlleven a los alumnos a planear, organizar, comprender y finalmente aprender. La pedagogía no es la enseñanza en sí, sino un saber sobre la misma (Ochoa et al., 2016).

Se define a la pedagogía como el conjunto de saberes que se encargan de la educación como fenómeno específicamente humano y típicamente social. Con lo cual podemos decir que, la pedagogía es aquella disciplina que regula el aprendizaje en el proceso educativo, se ocupa de los saberes orientados a la enseñanza-aprendizaje y abarca todas las ciencias educacionales, las metodologías y las técnicas que se utilizan durante la formación del ser humano.

La pedagogía, al presentarse como una ciencia, será observada, comprobada y, por ende, aceptada. De este modo, la pedagogía presenta las siguientes características: 
- Castillo (como se citó en López et al., 2017) expresa que la pedagogía tiene como característica clave la intervención docente, porque tiene el conocimiento del contenido a tratar en base al nivel y cátedra de estudio (pág. 15).

- Tiene como objetivo la construcción de una mejor ciudadanía, conscientes de su responsabilidad con su entorno.

- La pedagogía tiene diversos tipos que van desde la pedagogía conceptual, social hasta la pedagogía crítica, todas ellas corresponden a una mayor profundización del proceso de enseñanza-aprendizaje.

- Suárez et al. (2010) aseguran que la pedagogía "enfatizar en el desarrollo de las habilidades cognitivas" (pág. 93).

- La pedagogía “posee su campo intelectual de objeto y metodología de investigación propia, según cada paradigma o modelo pedagógico.”

\section{Pedagogía e interacción social}

En las internaciones sociales pedagógicas, en las experiencias sociales, se pueden observar o percibir, enfatizar el discurso explicito e implícito sobre las relaciones de los individuos. Es en esta relación donde se producen los comportamientos, conflictos he influencia sociales y culturales, desde esta perspectiva, es posible detectar la intencionalidad del comportamiento de interacción de los jóvenes y grupos sociales, en el marco de la práctica educativa.

En este caso del análisis específico, tomando en cuenta la experiencia del aula, puede resultar muy buena para comprender las aplicaciones de la pedagogía social que los estudiantes pueden tener para las internaciones en el aula y el papel de la pedagogía que los profesores ponen en práctica. Este análisis es, por tanto, a partir de las observaciones sistematizadas y entrevistas en profundidad para comprender la realidad en el aula en las condiciones actuales.

\section{Inteligencias múltiples y la pedagogía}

La pedagogía recibe influencias de diversas ciencias, como la psicología, la sociología, la antropología, la filosofía, la historia, la medicina, entre otras, para lograr comprender, explicar y guiar el proceso educativo, pero es la psicología la que tiene mayor importancia en la pedagogía, puesto que, esta le permite estudiar y comprender la mente, la conducta y forma de aprender de los individuos. Las ocho inteligencias definidas por Gardner se desarrollan de manera implícita en la pedagogía e influyen en gran medida en el desarrollo del proceso educativo (Chura, 2019).

Herrán (2018) refiere que en la actualidad la pedagogía tiene como objetivo el desarrollo de métodos y el establecimiento de técnicas óptimas de enseñanza- 
aprendizaje, que permitan desarrollar e incluir las distintas formas de aprender de los individuos, puesto que, durante el estudio de la educación se ha logrado comprender que no todos aprenden de la misma manera, sin embargo, lograr un aprendizaje significativo en el que se tome en cuenta las inteligencias múltiples se ha convertido en el gran reto.

\section{La teoría de las inteligencias múltiples y el proceso de enseñanza-aprendizaje}

Según Gardner (como se citó en Suárez et al., 2010), la teoría de las inteligencias múltiples simula señalando que cada inteligencia funciona como un computador que trabaja de manera independiente dependiendo del área en el que la persona se destaque, las inteligencias trabajan o se desarrollan en relación con las habilidades del individuo. Se dividen en las inteligencias verbales y escritas que se caracterizan por el habla, la inteligencia relacionada con la información espacial y entorno.

Según Mora (como se citó en Suárez et al., 2010) en el proceso educativo se deben evaluar al docente, currículo y metodología aplicada en la clase y la comunidad educativa. Las inteligencias en el ámbito educativo representan la capacidad de la persona al desenvolverse en diversas situaciones óptimas y en su desarrollo cognitivo. Los estudiantes aprenden a su propio ritmo y Gardner afirma, que no siempre aprendemos todo lo que se debe aprender, sino lo necesario para el desarrollo.

La inteligencia es una actividad psíquica compleja en la que se desarrolla la organización de nuestras habilidades para poder enfrentar situaciones problemáticas con la implementación de nuevos recursos o procedimientos y así resolver dichas situaciones de manera eficaz y eficiente (Chura, 2019).

Se conocen diferentes competencias en las que el ser humano puede desenvolverse, dependiendo de la categoría, que son: auditivo-oral, en la que se desarrollan inteligencias como: musical y lingüístico-verbal; la relación con los objetos se encuentran las inteligencias como: lógico-matemática, espacial, corporal-kinestésica, naturalista; finalmente encontramos la esfera de inteligencias personales, en las que se encuentran las inteligencias intrapersonales e interpersonales (De la Calle \& Aguilar, 2016).

Goleman (2013), afirma que "tenemos dos mentes, una que piensa y otra que siente". Las emociones son una de las facetas de ese proceso, una parte tan integral del mismo como el pensamiento lógico, lineal y verbal del hemisferio izquierdo. De la misma manera que no pensamos sólo con un único hemisferio, sino que los dos son necesarios.

Pedagogía es el conjunto de disciplinas desarrollada con autonomía funcional, realiza una valoración en el ámbito educativo, esta denominado como una ciencia de educación y un arte, donde se promueve la reflexión, análisis, estructura y adapta cada uno de los conocimientos adquiridos a través del tiempo (Herrán, 2018). 
La pedagogía involucra a las inteligencias múltiples dentro del sistema educativo, sin embargo, en la actualidad, el modelo educativo no incluye el desarrollo de estas en sus currículos. Macías et al., (2020), mencionan que "se manifiesta en la presente sistematización de ideas, metodologías y técnicas que promueve mentes creadoras e innovadoras. Como lo indica López et al. (2017) "el proceso de analizar, entender y evaluar forma parte del pensamiento crítico" (p.88).

Entre los estudios, adecuaciones y certezas en la aplicación de las inteligencias múltiples, se comprueba que cada ser humano reacciona individualmente ante los estímulos del contexto sociocultural afectivo, es por ello por lo que, en el sistema educativo, que se convierte en inteligencia ponderada, según sea el caso, y es necesario reforzar actividades y recursos pedagógicos que fortalezcan las áreas linguiísticas, motrices, de razonamiento, entre otras (Macías et al., 2020).

La aplicación de esta teoría ofrece beneficios positivos, ahora bien para esta aplicación se resaltará que en casos reales es necesaria una planificación de cómo se llevará a cabo el su desarrollo ya que no siempre va a estar presente esta teoría, se da por etapas y solo se aplican una o dos veces en todo el proceso, los resultados han sido favorables pues se han aumentado los porcentajes de manera significativa, proporcionando mayor eficacia en el aprendizaje autónomo, la inteligencia se desarrolla y acomoda al criterio del estudiante, a su vez se adapta a un ritmo natural, no forzado ni copiado, pues la manera de aprender de cada uno es distinta (Paida, 2018).

\section{Metodología}

La investigación se ejecutó a partir de la revisión documental y bibliográfica de artículos científicos publicados desde enero del 2016 hasta noviembre del 2021, en las bases de datos regionales: Latindex, Redalyc, Scielo, así como Scopus. La búsqueda se realizó mediante PubMed y se han clasificado por dos objetivos de búsqueda con los descriptores o palabras claves: inteligencias múltiples, pedagogía, didáctica, aprendizaje, desarrollo cognitivo.

Se identificaron los estudios o investigaciones en los que se abordaron inteligencias múltiples y la forma en que debe ser estudiado en los procesos pedagógicos, es decir, con el proceso de aprendizaje, los mismos que se han desarrollado en diferentes países de América del Sur. Se trabajó con nueve artículos todos con metodología cualitativa en los que aplicaron algunas técnicas de análisis bibliográfico, así como de aplicación estadística.

Criterios de inclusión: Fueron investigaciones publicadas entre 2016 y 2021 en países de américa del sur en idioma español. 
Criterios de exclusión: Estudios en los que no se incluya las inteligencias múltiples como parte del proceso de aprendizaje.

La investigación busca actualizar conocimientos relacionados con las inteligencias múltiples y su relación con los procesos de aprendizaje, especialmente, en estudiantes; es un estudio de revisión sistemática, no se han efectuado aplicación y tampoco intervención, es decir, se convierte en una compilación de información para marcar el punto de partida de nuevas investigaciones que permitan la profundización de la temática.

Una vez establecido el listado se seleccionaron los artículos que tratan o analizan las inteligencias múltiples, el desarrollo cerebral y el proceso de aprendizaje.

\section{Resultados}

Se identificaron publicaciones científicas que tratan o analizan las inteligencias múltiples, pedagogía, didáctica, aprendizaje, desarrollo cognitivo. Con esa especificidad se encontraron nueve artículos en los que aplicaron enfoque cualitativo y cuantitativo, a partir de revisión bibliográfica y documental, así como estudios de campo basados en encuestas.

Según Chura (2019), la teoría de las inteligencias múltiples de Howard Gardner en la pedagogía logra mayor atención, porque con el paso del tiempo se han aplicado en diferentes programas educativos y que se han convertido en la base de estudios de psicología educativa, encargada de abordar los problemas que presenta la pedagogía para diseñar métodos y establecer técnicas psicológicas que permitan lograr óptimos niveles de aprendizajes y desarrollo de las diversas capacidades de los estudiantes.

El estudio analiza la relación vigente entre las concepciones de la inteligencia y la pedagogía y mostrar la evolución en conjunto. En el mismo se sustenta que la inteligencia es el proceso psicológico cognitivo superior, que usa capacidades y habilidades del sujeto para solucionar problemas nuevos de manera eficiente. Entre los resultados alcanzados, señalan que la facultad del ser humano que le permite adaptarse a diferentes entornos y circunstancias, donde el sujeto usará sus cualidades psicológicas aprendidas para resolver con éxito distintos problemas o determinadas actividades. Contreras (2013), señala que la inteligencia como el uso de las habilidades y capacidades para resolver problemas que están inmersos en el ambiente. En ese contexto Chura (2019), señala que la inteligencia es una actividad psíquica relacionado con el éxito de su paso por la escuela, el tipo de trabajo que realizamos, de recreación e incluso en la elección de los amigos. 
Chura (2019), indica que la inteligencia es la capacidad que tiene el hombre para razonar, entender y juzgar debidamente, aspectos contemplados en el proceso de aprendizaje, por lo tanto, capaz de resolver problemas y sobre todo que hoy en día la psicología educativa investiga sobre el aprendizaje y la enseñanza, al mismo tiempo, trabaja para mejorar la práctica educativa.

Algunas hipótesis y las demuestra fundamentado en la aplicación de la ficha de observación y una encuesta a niños, mostrando una relación estadística significativa y positiva entre las variables analizadas. Uno de los resultados cotejada con la discusión determina que las ocho inteligencias múltiples se registran en los estudiantes de forma implícita, por lo que la educación debe tener un carácter holístico que incluya lo cognitivo y conceptual, así como las dimensiones sociales, emocionales, afectivas y motoras.

Se investiga la relación entre las variables con la aplicación de una entrevista de profundidad y la ficha de observación, instrumentos cuyos resultados revelan que las inteligencias están asociadas con las condiciones sociales y culturales de las comunidades. Los datos registrados de la aplicación se describen y contrastan utilizando la fenomenología, es decir, el detalle pormenorizado de la mencionada relación.

Tabla 1

Resumen de artículos relacionados con inteligencias múltiples

\begin{tabular}{|c|c|c|c|c|}
\hline Año & Autor & País & Objetivo & $\begin{array}{l}\text { Enfoque metodológico (Enfoque } \\
\text { técnica, instrumentos) }\end{array}$ \\
\hline 2019 & Chura & Perú & $\begin{array}{l}\text { Determinar las bases } \\
\text { epistemológicas de la teoría de las } \\
\text { inteligencias múltiples de Howard } \\
\text { Gardner en la pedagogía }\end{array}$ & $\begin{array}{l}\text { Cualitativo de tipo teórico- } \\
\text { hermenéutico con un alcance } \\
\text { correlacional a través del análisis } \\
\text { de teorías y contenidos } \\
\text { relacionados a las inteligencias } \\
\text { múltiples y la pedagogía. }\end{array}$ \\
\hline 2001 & Gardner & España & $\begin{array}{l}\text { Entender y reconocer las } \\
\text { diferentes inteligencias y } \\
\text { diferentes ejercicios para pulirlas } \\
\text { de manera autónoma. }\end{array}$ & $\begin{array}{l}\text { Enfoque cualitativo. Ha utilizado } \\
\text { métodos de nivel teórico- } \\
\text { histórico, inductivo deductivo y } \\
\text { del nivel empírico el análisis de } \\
\text { documento. }\end{array}$ \\
\hline 2018 & $\begin{array}{l}\text { Calder, } \\
\text { Llamas, } \\
\text { Salguero \& } \\
\text { López. }\end{array}$ & España & $\begin{array}{l}\text { Analizar las relaciones existentes } \\
\text { entre las variables } \\
\text { neuropsicológicas: inteligencias } \\
\text { múltiples, rendimiento académico } \\
\text { y creatividad. }\end{array}$ & $\begin{array}{l}\text { Enfoque mixto a partir de la } \\
\text { aplicación de diferentes técnicas } \\
\text { como observación y encuesta a } \\
\text { niños de } 7 \text { a } 11 \text { años. }\end{array}$ \\
\hline
\end{tabular}




\section{Tabla 1}

\section{Resumen de artículos relacionados con inteligencias múltiples (continuación)}

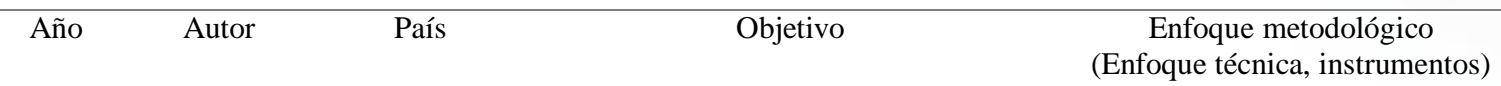

\begin{tabular}{|c|c|c|c|}
\hline 201 & $\begin{array}{l}\text { Peña, } \\
\text { Ezquerro, } \\
\text { López, et al. }\end{array}$ & Colombia & $\begin{array}{l}\text { Analizar la relación entre Creatividad, } \\
\text { Inteligencias Múltiples y Rendimiento } \\
\text { Académico en estudiantes de Básica. }\end{array}$ \\
\hline
\end{tabular}

Cualitativo no experimental descriptivo con alcance correlacional. Estudio sobre la población de 40 sujetos 20 niños y 20 niñas del Municipio de Yaguará-Colombia

\begin{tabular}{|c|c|c|c|}
\hline 2018 & $\begin{array}{l}\text { Peña- } \\
\text { Rodríguez, } \\
\text { M.A.et al. }\end{array}$ & México & $\begin{array}{l}\text { Comprender las inteligencias en cada } \\
\text { entorno, las estrategias didácticas que } \\
\text { aplican los maestros, las inteligencias } \\
\text { que se favorecen de acuerdo con las } \\
\text { características socioculturales y con } \\
\text { objetivos de formación }\end{array}$ \\
\hline
\end{tabular}

\section{Cualitativo con diseño} fenomenológico, en base a la aplicación de entrevistas semi estructuradas, así como ficha de observación

\begin{tabular}{|c|c|c|c|c|}
\hline 2021 & $\begin{array}{l}\text { Tobón, } \\
\text { Marín, } \\
\text { Llanos et al. }\end{array}$ & Colombia & $\begin{array}{l}\text { Diseñar una estrategia didáctica de } \\
\text { aprendizaje para los tipos de } \\
\text { inteligencias predominantes. }\end{array}$ & $\begin{array}{l}\text { Cualitativo aplicado a } \\
\text { estudiantes de quinto grado de } \\
\text { educación básica en base a } \\
\text { observaciones. }\end{array}$ \\
\hline 2016 & $\begin{array}{l}\text { Castillo, } \\
\text { Ezquerro \& } \\
\text { López et al. }\end{array}$ & España & $\begin{array}{l}\text { Analizar la relación estadísticamente } \\
\text { significativa entre la creatividad y cada } \\
\text { una de las inteligencias múltiples de la } \\
\text { muestra; ratificar de forma empírica la } \\
\text { relación entre los procesos cognitivos } \\
\text { implicados en tareas de creatividad y las } \\
\text { funciones ejecutivas. }\end{array}$ & $\begin{array}{l}\text { Enfoque cuantitativo con la } \\
\text { aplicación de cuestionario de } \\
\text { Inteligencias Múltiples de } \\
\text { Armstrong, así como la prueba } \\
\text { de diagnóstico de creatividad de } \\
\text { Marín, que se aplicó a } 29 \text { niños } \\
\text { de entre } 8 \text { y } 10 \text { años. }\end{array}$ \\
\hline 2020 & Macías et al. & Cuba & $\begin{array}{l}\text { Evidenciar las estrategias metodológicas } \\
\text { interoceptivas y exteroceptivas de las } \\
\text { inteligencias múltiples en el proceso de } \\
\text { enseñanza- aprendizaje, según su propio } \\
\text { estilo, y proponer una guía dialógica } \\
\text { inferencial argumentativa de estas ocho } \\
\text { inteligencias, como aporte académico a } \\
\text { los docentes del contexto manabita, } \\
\text { ecuatoriano y mundial. }\end{array}$ & $\begin{array}{l}\text { Enfoque cualitativo se basa en } \\
\text { la interpretación de hechos tras } \\
\text { un estudio por medio de } \\
\text { técnicas para lo que se aplicó la } \\
\text { observación participante a la } \\
\text { población definida de entre } 9 \text { y } \\
12 \text { años. }\end{array}$ \\
\hline 2020 & Mena et al. & Brasil & $\begin{array}{l}\text { Indagar las inteligencias múltiples } \\
\text { predominantes en estudiantes de } \\
\text { ingeniería }\end{array}$ & $\begin{array}{l}\text { Enfoque cuantitativo, en base a } \\
\text { una encuesta sobre la población } \\
\text { de } 7798 \text { estudiantes, la cual fue } \\
\text { respondida por } 1727 \text { de las } \\
\text { facultades de Ciencias, } \\
\text { Biofísica, Estadística, } \\
\text { Informativa, Física y } \\
\text { Matemática, Control y Redes, } \\
\text { Telecomunicaciones, } \\
\text { Electrónica y Automatización y } \\
\text { Sistemas, automotriz, } \\
\text { Mantenimiento e Industrial }\end{array}$ \\
\hline
\end{tabular}


Se trazaron para esta investigación objetivos muy concretos, recolectaron la información derivada de las respuestas a los instrumentos (encuestas) aplicados a las tres fuentes de información escogidos entre ellos, docentes, sobre aspectos específicos: Estrategias didácticas de aprendizaje, que se vuelven elementos propositivos e intencionados con los que cuenta un docente para encaminar la enseñanza directamente hacia el aprendizaje significativo por medio de los recursos dispuestos en el contexto educativo, la experiencia con que cuenta y las características particulares de los estudiantes y lo que estos como grupo llegan a representar.

Así también señalan sobre los recursos educativos, que la preferencia de la mitad de los docentes está orientada hacia el uso de las tecnologías de información y la comunicación. Otro opta por el uso de material físico y el último, se inclina por la combinación de ambos recursos, lo que significa que, en general, los profesores están alineados con las exigencias del contexto educativo actual y las condiciones sociales en las que están inmersos los estudiantes. De la misma manera, este grupo de maestros, en el uso de los elementos didácticos, trabaja de la mano con lo que hay a su disposición por parte de la institución enlazados con los objetivos del aprendizaje.

Por su parte Castillo, Ezquerro \& López aplicaron el cuestionario de Inteligencias Múltiples de Armstrong, así como la prueba de diagnóstico de creatividad de Marín, a 29 niños de entre 8 y 10 años. Los resultados señalan que la estimulación de las inteligencias múltiples abarca la fundamentación, planificación, contextualización y sistematización curricular lúdica -afectiva - pedagógica y que finalmente, se logra un ser humano más integral, solidario, académico, existencial, propositivo y productivo en la sociedad cambiante, diverso en donde se desenvuelva con una proyección universalista.

Macías et al. (2020), basaron su investigación en la técnica de la observación participante a la población de estudiantes de entre 9 a 12 años. Los resultados muestran una correlación positiva y estadísticamente significativa entre las inteligencias múltiples y el rendimiento académico. Además, amplían el análisis en base a las hipótesis planteadas en comparación con los estudios previos identificados en el marco teórico. Según el estudio, a teoría de las inteligencias múltiples deja como legado a la comunidad académica la metacognición en el campo educativo. Su reconocimiento corrobora la consolidación de procesos enriquecidos con el entorno afectivo, la disciplina positiva, la resiliencia desde la familia y el reforzamiento de esta en la escuela innovadora y diferente, por lo que promueven nuevas alternativas que mejorarán el desempeño del docente.

La aplicación de la encuentra y la ficha de observación a niños de 7 a 11 años permitieron establecer relaciones entre las dos variables estudiadas. Uno de los resultados más significativos es que implementación de la teoría de las inteligencias 
múltiples de Gardner genera beneficios sobre la atención de las individualidades de los estudiantes y los aprendizajes.

Finalmente, Gardner (2001), en su investigación utiliza métodos de nivel teóricohistórico, inductivo deductivo y del nivel empírico el análisis de documentos y concibe como resultados que hay ocho inteligencias y que existen varios ejercicios para identificarlas. Para sustentar los beneficios de la aplicación de la teoría de Gardner en las distintas actividades desarrolladas, evaluaron a los estudiantes tanto en la primera como en la segunda lección. En el primer año de educación general básica se evalúa la manera cualitativa para verificar y favorecer el desarrollo integral de niños, sin que exista un proceso de calificación paralela.

Este estudio también se realiza con la aplicación de la escala propuesta por el Ministerio de Educación en el Ecuador, logrando algunos resultados coherentes con la existencia de las inteligencias múltiples, como que los estudiantes adquirieron la destreza de identificar semejanzas y diferencias entre objetos.

Para Mena et al. (2020), se trabajó siete dimensiones para la encuesta con la escala de Likert a, la misma que agrupó a en variables, el procedimiento consistió en crear una dimensión en el que aparecen los resultados con los que se elaboró la tabla de contingencia. En la misma aparecen las varias inteligencias con sus respectivos porcentajes, en la que concluyen que en tres facultades la que menos predomina es la naturalista, en electrónica la que predomina es la musical, en mecánica la intrapersonal; en redes y telecomunicaciones predomina la lógica matemática

\section{Conclusiones}

- La teoría elaborada y probada sobre las inteligencias múltiples por Howard Gardner sin duda ha revolucionado los conceptos tradicionales incorporando de manera muy directa su aplicación en los procesos educativos. En Ecuador incluso han sido analizadas con diferentes objetivos.

- El ámbito de la pedagogía y su relación directa con la teoría de las inteligencias múltiples desarrollada desde el punto de vista multifacético y evidenciado en ocho diferentes y a la vez interconectadas entre sí, confirma que existe una interdependencia que se evidencia con mayor profundidad en el campo de la educación y el desarrollo de su perfil cognitivo.

- Una vez identificadas las características de cada una de las ocho inteligencias identificadas en cada uno de los hemisferios cerebrales, se han promovido diferentes programas o posibilidades metodológicas que contribuyan al desarrollo de unas u otras, sin descuidar el andamiaje integral de la enseñanza aprendizaje. 
- Resulta imponderable que el sistema educativo ecuatoriano trate de incorporar nuevas metodologías y prácticas didácticas para potenciar las diferentes inteligencias que se desarrollan en los individuos y desde allí trabajar los talentos, especialmente, en el ámbito de las artes, donde tanto Ecuador y toda América del Sur, tienen déficits.

- Es determinante el uso de la tecnología, que en la actualidad está inmerso en todos los ámbitos, por lo tanto, la teoría de las inteligencias múltiples, también debe considerarla a la hora de aplicar estrategias pedagógicas.

\section{Referencias bibliográficas}

Chilig, H. (2018). Inteligencias http://www.dspace.uce.edu.ec/bitstream/25000/17246/1/T-UCE-0010-FIL204.pdf

Chura, E. (2019). Bases epistemológicas que sustentan la teoría de las inteligencias múltiples de Howard Gardner en la pedagogía. Innova Educación. 590-598. http://www.revistainnovaeducacion.com/index.php/rie/article/view/62

Contreras, L. C. (2013). La inteligencia intuitiva y la educación en creatividad. Actualidades $\quad$ Pedagógicas, (62), 165-187. Doi: https://doi.org/10.19052/ap.2697

De la Calle, A. \& Aguilar, M. (2016). Desarrollo evolutivo de la conciencia fonológica: ¿Cómo se relaciona con la competencia lectora posterior? Revista de Investigación en Logopedia. 22-41

Gardner, H. (2001). Estructuras de la mente: La teoría de las inteligencias múltiples http://biblioteca.udgvirtual.udg.mx:8080/jspui/bitstream/123456789/593/1/Estru ctura\%20de\%20la\%20mente.\%20teoria\%20de\%20las\%20Inteligencias\%20mult iples.pdf

Ernest-Slavit, G. (2001). Educación para todos: La Teoría de las Inteligencias Múltiples de Gardner. Revista de Psicología de la PUCP 29 (3), 320-333. https://doi.org/10.18800/psico.200102.006

Goleman. (1996). Inteligencia emocional. Editorial Kairos

Goleman. (2013). El cerebro y la inteligencia emocional: Nuevos descubrimientos. https://bibliotecaia.ism.edu.ec/Repo-book/e/El-cerebro-y-la-inteligenciemocionalDanielGoleman.pdf 
Herrán Gascón, A. (2018). Para una pedagogía futura más compleja y consciente. Apuntes de Pedagogía, 24-26.

Jensen, E. (2010). Cerebro y aprendizaje competencias e implicaciones educativas. Madrid, España. NARCEA, S.A. de Ediciones.

López, R., Benítez, R., \& Garcias, M. (2017). Caracterización pedagógica de los componentes y elementos, del diseño tutorial de cursos a distancia sobre plataformas gestoras. Revista Científica de la Universidad de Cienfuegos, 13-18.

Macías, Y., Vigueras, J., Rodríguez, M. (2020). Una escuela con inteligencias múltiples: visión hacia una propuesta innovadora. Revista cubana de Educación Superior.

Mena, A., Tuapanta, J. \& Santillán, J. (2020). Análisis exploratorio sobre las inteligencias predominantes en los estudiantes de ingenierías de la Escuela Politécnica de Chimborazo. Espacios.

Nadal, B. (2015). Las inteligencias múltiples como una estrategia didáctica para atender a la diversidad y aprovechar el potencial de todos los alumnos. Revista nacional e Internacional de Educación Inclusiva, 121-136

Ochoa, M., Caballero, C., Moreno, A., \& Valcárcel, N. (2016). Pensamiento crítico en acción: Cooperación Genuina como aporte en la construcción de escenarios educativos alternativos. "ABACO en Red", 1-152.

Paida, A. M. (05 de 10 de 2018). La teoría de las inteligencias múltiples en la educación. https://polodelconocimiento.com/ojs/index.php/es/article/view/732/907

Prado Yépez, L. E., Viteri Gordillo, M. J. \& Rojas Núñez, M. del P. (2017). Aporte del pensamiento lateral al desarrollo de la inteligencia lingí1/4í-stica. Revista Publicando, $4(13(2)$, 269-281. https://revistapublicando.org/revista/index.php/crv/article/view/862

Ramírez, R. (2018). Inteligencias Múltiples En El Trabajo Docente y su relación con la Teoría del Desarrollo Cognitivo de Piaget. Revista Killkana SocialesUniversidad Católica de Cuenca. 47-52

Suárez, J., Maíz, F., \& Meza, M. (2010). Inteligencias múltiples: una innovación pedagógica para potenciar el proceso de enseñanza aprendizaje. Investigación y Postgrado, 81-94 


\section{DDigital}

www.concienciadigital.org

Valbuena-Duarte, S., Padilla Escorcia, I. \& Rodríguez Bossio, E. (2018). Reconocer la inteligencia lógico-matemática en estudiantes con capacidades excepcionales. Tecné, Episteme y https://revistas.pedagogica.edu.co/index.php/TED/article/view/8152

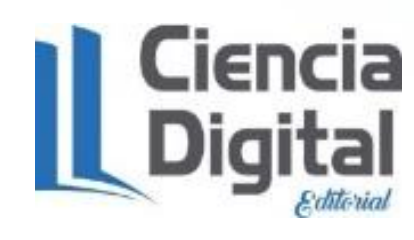


El artículo que se publica es de exclusiva responsabilidad de los autores y no necesariamente reflejan el pensamiento de la Revista Conciencia Digital.

\section{Ciencia \\ LDigital}

El artículo queda en propiedad de la revista y, por tanto, su publicación parcial y/o total en otro medio tiene que ser autorizado por el director de la Revista Conciencia Digital.
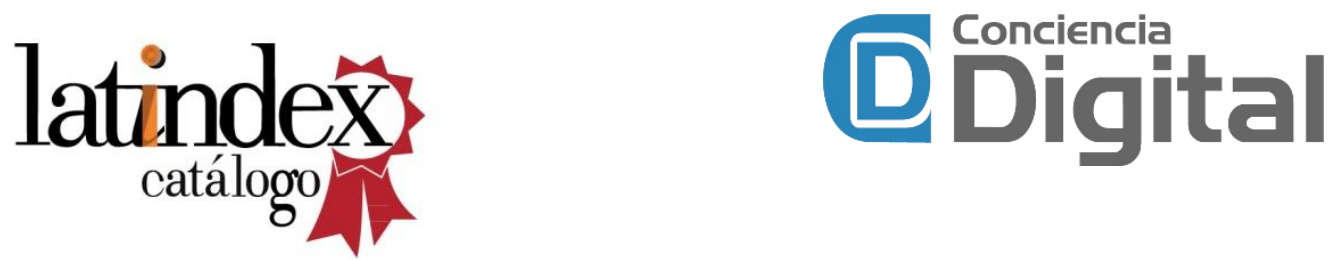

Indexaciones

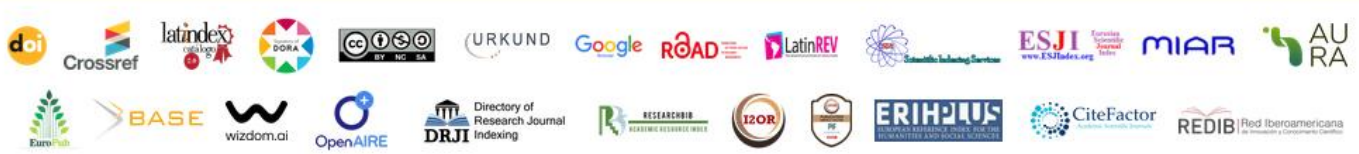

